Version of Record: https://www.sciencedirect.com/science/article/pii/S1572665719302085

Manuscript 2b68a83d134300049864fa5d40d669ab

\title{
Extrapolation to zero frequency with first time constant of ferri-ferrocyanide/Pt interface using Foster equivalent circuit and factorised modelling
}

\author{
P. LAGONOTTE, V.A. RAILEANU ILIE, S. MARTEMIANOV, A. THOMAS* \\ Institut Pprime, Université de Poitiers-CNRS-ENSMA, UPR 3346, 2 Rue Pierre Brousse, Bâtiment B25, TSA \\ 41105, F-86073 Poitiers Cedex 9, France.
}

\begin{abstract}
:
An estimation of low frequency parameters at the interface of a ferri-ferrocyanide solution on a Pt rotating disk electrode is presented. The determination of the total resistance and effective (low frequency) capacity is based on a Foster's model extrapolation using electrochemical impedance spectroscopy measurements. The feasibility of the proposed approach has been demonstrated at equilibrium conditions and under two polarization points. Results show a good agreement with the identified and experimental values particularly in the extra-low frequency domain. Results also demonstrate that using factorized expression (poles and zeros) of the impedance allow the stability of the identified parameters and the calculation of the first time-constant of the system. Consequently, Foster's models as equivalent to Voight-type equivalent circuit can be used for the zero frequency extrapolation and predict the response of the interface in time domain that it is certainly useful for practical electrochemical systems.
\end{abstract}

Keywords: Identification, Foster's modelling, zero frequency extrapolation, Low-frequency parameters, DC resistance, First-time constant, ferri-ferrocyanide, Electrochemical systems

*corresponding author: anthony.thomas@univ-poitiers.fr

\section{Introduction}

Electrochemical impedance spectroscopy (EIS) is one of the most powerful, and usual method to study electrochemical systems and interfaces [1,2]. EIS is a truly sensitive method but the complexity of electrochemical phenomena makes difficult the adequate choice of models which can be used for description of electrochemical systems and even relatively simple interfaces. Sure, this is the problem for using EIS. Indeed, in practical applications it is not difficult to reach a good concordance between experimental impedance spectra and its numerical simulation using fitting parameters of the equivalent model. Nevertheless, this concordance does not justify the validity of the using model and further interpretation of the obtained numerical results is an open question.

Numerous models can be associated with EIS measurements. In general, any impedance spectra can be rationalized in terms of finite or infinite RC parallel or serial (Foster) or ladder (Cauer). Regarding solid / liquid interface, all these models meet often a problem of physical justification. For example, at an interfacial electrochemical reaction it is often difficult to obtain a quantitative description and phenomenological models are usually used to interpret the data. The using of a constant phase element (CPE) in equivalent circuits [1] allows efficient fitting of experimental impedance data but leaves open the question of interpretation [3-6].

In a case of phenomena are strictly govern by diffusion effect, the physic can be described using mass transport equations and interpreted in terms of Warburg impedance. In this case the Foster and Cauer model are not strictly applicable because Warburg element is equivalent to infinite number of resistance and capacity.

Consequently, a good fitting of experimental data can be reached using different equivalent circuits [7] and associated with these models different equivalent electrical circuits [8,9]. Increasing the number of elements improves the fitting but very often perturbs previously obtained model parameters. Sure, only the parameters which are stable with respect to model perturbations are suitable for physical interpretation. The criterion of stability seems to be more important than a simple improvement of fitting when one looks for physical interpretation of model parameters. 
Moreover, the utility of the model depends on it's predictively. Indeed, only the model allowing extraction of additional information from experimental data are useful for practical applications. Thus, a good fitting of experimental data, the stability of identified parameters regarding to the model perturbations and the predictively justify the selection of one model to another for the interpretation of impedance data by means of equivalent electrical circuits.

The present paper deals with interpretation of impedance measurements by means of Foster's models [9]. Foster's models present one of the possible topologies based on RC series (Fig. 1). Although Foster model is an approximation of Warburg element, this allows to determine the high frequency resistance $\left(\mathbf{R}_{\mathbf{H F}}=\mathbf{R}_{\mathbf{0}}\right)$ and the total ( $\mathbf{R}_{\boldsymbol{\Sigma}}-\mathrm{dc}$ resistance) of solid-liquid interface by an extrapolation to zero frequency, especially in the case where the zero frequency tends to a vertical asymptote. Identification of the total resistance is not a trivial problem due to the difficulty of impedances measurements in low frequency range $(\boldsymbol{f} \rightarrow \mathbf{0})[10,11]$. Using Foster's models gives a possibility to replace long-term low frequency measurements by a fast modelling response that can be useful for practical applications. Another important point concerns possibility of determining time constant phenomena and effective capacity of solid / liquid interface.

\section{Material and methods}

All electrochemical measurements were carried out in a conventional two-electrode cell. The working electrode is a platinum disk embedded in PTFE support with a diameter of $2 \mathrm{~mm}$, for a surface of $0.03 \mathrm{~cm}^{2}$ and a platinum slab of $2 \mathrm{~cm}^{2}$ is used as counter electrode. The electrolyte consisted of deionized water and an equimolar $0.025 \mathrm{M}$ potassium ferricyanide $\mathrm{K}_{3} \mathrm{Fe}(\mathrm{CN})_{6}$, and $0.025 \mathrm{M}$ potassium ferrocyanide $\mathrm{K}_{4} \mathrm{Fe}(\mathrm{CN})_{6}$. Potassium sulphate $\mathrm{K}_{2} \mathrm{SO}_{4}$ was used as the supporting electrolyte at concentrations of $0.3 \mathrm{M}$. Experiments were performed at $20^{\circ} \mathrm{C}$ in a rotating disk electrode (RDE) configuration. The electrochemical experiments were repeated at least three times to ensure that obtained data are reproducible. All electrochemical experiments were carried out using Solartron Multistat 1480 potentiostat and EIS a Solartron Frequency Analyser 1260. EIS experiments were carried out by applying a sinusoidal potential wave with amplitude of $6 \mathrm{mV}$. The impedance response was measured over frequencies between $1 \mathrm{MHz}$ and $0.1 \mathrm{~Hz}$.

Fig. 2 presents the obtained current-voltage curve with three operating points where impedance measurements have been performed, namely $\mathrm{I}=0 \mu \mathrm{A}$ (equilibrium), $\mathrm{I}=100 \mu \mathrm{A}(\mathrm{V}=0.02 \mathrm{~V})$ and $\mathrm{I}=200 \mu \mathrm{A}(\mathrm{V}=$ $0.05 \mathrm{~V})$. From Fig. 2 the calculation of the dynamic resistance $\mathrm{R}_{\text {dyn }}$ can be done considering small variations $\Delta \mathrm{V} / \Delta \mathrm{I}$ around studied point. The results of calculations are presented in Table 1 and will be used later for comparison with impedance data.

Figure 3 presents the result of impedance measurements for these selected points. It can be seen an important phase dependence on the frequency and existence of two arcs. The first arc in high frequency region is often associated to charge transfer phenomena and the second arc to the diffusion effect. In high-frequency range the intersection of the impedance curve with axe $Z$ ' $=0$ allows determining high-frequency resistance $\mathbf{R}_{\mathbf{H F}}=\mathbf{4 5} \Omega$. This resistance can be associated with the effective solution resistance and estimated using the well knows expression of Newman for a disc configuration [12]:

$$
\mathrm{R}_{\mathrm{disc}}=1 /(4 \sigma \mathrm{r})
$$

where $\sigma$ is the solution conductivity $\left(\mathrm{S} \cdot \mathrm{m}^{-1}\right)$ and $\mathrm{r}$ is the electrode radius (here $\mathrm{r}=0.001 \mathrm{~m}$ ).

Thanks to an electrical conductivity meter measurement, the solution conductivity is equal to $62.3 \pm 0.5$ $\mathrm{mS} . \mathrm{cm}^{-1}$ at $\mathrm{T}=20^{\circ} \mathrm{C}$ that implies a solution resistance of $\mathrm{R}_{\text {electrolyte }}=40.1 \pm 0.3 \Omega$ comparable to high frequency resistance $R_{H F}$ of $45 \Omega$. 
In low frequency range it can be noted that the impedance data are noisy-affected. Determination of lowfrequency resistance (total resistance) is not so evident and goes though extrapolation of extra-low frequency arc data to intersect Z" $=0$. All these methods involve some modelling which are accepted usually without discussion.

In the next section, the interpretation of impedance data is done using Foster's models approach. This approach allows estimating the stability of identified parameters with respect to the increasing of the number of model elements. After, the identified stable parameters are used for physical interpretation. Moreover, advantage of Foster's models, it that RC elements assume a simple transition from frequency to time domain that can be useful for practical issues.

\section{Results}

\subsection{Extrapolation of impedance data using Foster's models}

The topology of Foster's models used is presented on Fig.1. Identification of model parameters $\left[\mathrm{R}_{0}, \mathrm{C}_{1}\right.$, $\left.\mathrm{R}_{1}, \ldots, \mathrm{C}_{\mathrm{N}}, \mathrm{R}_{\mathrm{N}}\right]$ has been done by minimisation of error between experimental and numerical impedance data on Nyquist diagram using criteria with modulus weighting [13]:

$$
\text { Criteria }=\frac{1}{701} \sum_{n=1}^{n=701} \frac{\sqrt{\left|Z_{\text {measure }}-Z_{\text {model }}\right|^{2}}}{Z_{\text {measure }}}
$$

where $n=701$ corresponds to a total number of experimental points.

The results of identification for equilibrium point $(\mathrm{I}=0 \mu \mathrm{A})$ are summarized on Table 2 and for the points $\mathrm{I}=100 \mu \mathrm{A}, \mathrm{I}=200 \mu \mathrm{A}$ on Tables $\mathrm{A} 1$ and $\mathrm{A} 2$ in annexe 2.

Table 2 summarizes the results of identification for $\mathrm{I}=0 \mu \mathrm{A}$ and different number $\mathrm{N}$ of Foster's series.

It can be noticed an improvement of the fitting criteria from $\mathrm{N}=1$ to $\mathrm{N}=4$ and its stagnation from $\mathrm{N}=4$ to $\mathrm{N}=6$. So, Foster's model allows a good fitting of the impedance data in all experimental frequency range with $\mathrm{N}=4$, see Fig. 4.

Only two parameters are stable with respect to increasing of $\mathrm{N}$, namely the initial resistance $\mathrm{R}_{0}$ and the total resistance $\mathrm{R}_{\Sigma}$. These identified models parameters are suitable for physical interpretation.

The resistance $\mathrm{R}_{0}$ can be associated with the resistance of the working electrode (electrolyte resistance):

$$
R_{0} \approx 45.7 \Omega
$$

Its value is in a good agreement with presented above traditional identification of high-frequency resistance $\mathrm{R}_{\mathrm{HF}}$.

The total resistance $\mathrm{R}_{\Sigma}$ can be associated with low-frequency resistance:

$$
\mathbf{R}_{\Sigma} \approx 236 \Omega
$$

Its value is in a good agreement with the value identified after extrapolation of experimental data to extralow frequency domain. Other results are given on annexe 2.

It can be noted that for all studied experimental points a good fitting of experimental data are obtained with Foster's models starting from $\mathrm{N}=4$. The main part of identified parameters $(\mathrm{Rn}, \mathrm{Cn})$ is not stable with respect to the increasing of number of the elements $N$. Nevertheless, the initial resistance $R_{0}$ and the total resistance $\mathrm{R}_{\Sigma}$ are not sensitive to the increasing of $\mathrm{N}$ and can be identified as the physical parameters of solid / liquid interface. Table 3 presents these parameters for different experimental points. A good agreement between identified values of total resistance $\mathrm{R}_{\Sigma}$ and dynamic resistance $\mathrm{R}_{\mathrm{dyn}}$ calculated previously from the polarization curve is obtained. 


\subsection{Identification of characteristic times-constants of solid / liquid interface}

For practical applications, identification of time constants of the system is very important. Usually, the time constant of the system is determined as:

$$
\tau_{c h}=\frac{1}{R_{c h} \cdot C_{c h}}
$$

where $R_{c h}$ is a characteristic resistance and $C_{c h}$ is a characteristic capacity of the system.

Presented above, results show that Foster's models allow identification of high-frequency and total resistances of solid / liquid interface. The question of identification of a characteristic capacity from Tables 2, $\mathrm{A} 1, \mathrm{~A} 2$ is open, because all the identified capacities change drastically with $\mathrm{N}$.

Nevertheless, it is possible to solve this problem by introducing the factorized impedance of the identified circuit. The expressions of the factorized impedance are given on Fig. 5. Thus, there are two equivalent expressions of the Foster's impedance, namely one obtained by simple summarizing of the impedances of different stages (developed impedance) and the second factorized. Here $s=j \omega$.

To factorise the developed expression for Foster's impedance, the "factor" function of Maple ${ }^{\circledR}$ software is used. In this expression $\mathrm{P}_{\mathrm{n}}$ and $\mathrm{Z}_{\mathrm{n}}$ are poles and zeros of the factorized impedance functions. Characteristic time constants of the system are given by the values of poles and zeros [14], see also Annexe 1. Tables 4-6 present the values of calculated poles and zeros for three experimental points.

It can be noted that in the range $3 \leq \mathrm{N} \leq 6$, the first pole $\mathrm{P}_{1}$ can be identified with $5 \%$ of accuracy (see Table 4 to 6$)$.

\section{Discussion}

In previous sections, it has been demonstrated that using Foster's model allows good fitting of impedance data starting from $\mathrm{N}=4$ stages. Nevertheless, only few identified model parameters are stable with respect to increasing of $\mathrm{N}$ and can be used for physical interpretation. These stable parameters are resistances $\mathrm{R}_{0}, \mathrm{R}_{\Sigma}$ and first pole $\mathrm{P}_{1}$ in factorized expression for the impedance of identified Foster's model. The identified resistance $\mathrm{R}_{0}$ gives the same value as high-frequency resistance $\mathrm{R}_{\mathrm{HF}}$ which is determined by the intersection of the impedance curve with the real axis $(Z "=0)$. The identified total resistance $\mathrm{R}_{\Sigma}$ can be interpreted as a low frequency resistance. Its value is in a good agreement with the dynamic resistance $R_{\text {dyn }}$ obtained from the polarization curve. The first pole $\mathrm{P}_{1}$ can be interpreted as a first time-constant of the system, its value corresponds also to the frequency at the extremum of Z".

The main interest of identification via Foster's models deals with a possibility to predict the system behaviour in low frequency domain using experimental impedance data for high and moderate frequency ranges. This possibility is demonstrated on Fig. 4 where Foster's model allows system identification in low frequency range $(0<\mathrm{f}<0.1 \mathrm{~Hz})$ using impedance data obtained for $0.1 \mathrm{~Hz}<\mathrm{f}<1 \mathrm{MHz}$.

Further interpretation can be obtained using simplified model with one pole and zero, see Fig. 6.

In this model previously identified resistances $\mathrm{R}_{0}, \mathrm{R}_{\Sigma}$ are introduced. Calculation of the characteristic capacity $\mathbf{C}^{\prime}{ }_{1}$ is done using Eq. (3) and identified value of the first pole $\mathrm{P}_{1}$. The results obtained for three specified current values are presented in Table 7.

Fig. 7 illustrates the domain of applicability of the simplified one stage model. In low frequency range $f$ $<0.1 \mathrm{~Hz}$ this simplified model (factorised model) is in perfect agreement with identification obtained by using 
Foster's model with $\mathrm{N}=4$ stages. Thus, identified capacity $\mathbf{C}^{\prime} \mathbf{1}$ can be interpreted as low frequency effective capacity of the system. Its value is very different from double layer capacity which is negligible in low frequency range. The low frequency capacity is related with first time-constant $\tau_{1}$ and determines the system behaviour in time domain (see Annexe 1).

Thanks to the first time constant $\tau_{1}$ (table 8) it is possible to calculate to the frequency $\mathbf{F}_{\mathbf{c} 1}$ corresponding to the maximum of the imaginary value of EIS curve (Fig 7). It is remarkable that the time constant $\tau_{1}$ and the frequency $\mathbf{F}_{\mathbf{c}}$ are outstandingly stable with respect to polarization, whereas the values $\mathbf{R}^{\prime} \mathbf{1}$ and $\mathbf{C}_{\mathbf{1}} \mathbf{1}$ vary.

In this experiment, the low frequency impedance spectrum of a charge transfer on an RDE is a semicircle approaching the abscissa vertically. In this case, the dominant RC gives good extrapolation to zero frequency. However, this method cannot be generalized to systems that do not approach the abscissa vertically.

\section{Conclusions}

Foster's models, as equivalent to Voight-type equivalent circuit, are useful for interpretation of impedance data, especially for extrapolation of the low frequency range ( $\mathrm{f}<1 \mathrm{~Hz}$ ). In particular, these models allow the identification of total resistance, low frequency capacity of the system. The feasibility of the proposed approach has been demonstrated using classical ferri-ferrocyanide electrochemical system in equilibrium conditions and under two polarization points. The main criteria allowing identification of physical parameters is the stability of these parameters with respect to model perturbations (increasing a number of stages). The parameters which do not meet this requirement should be considered as auxiliary model parameters. In particular, this is a case of all directly identified capacities presented in Foster's models.

The first time constant response of the interface has been determined using factorized modelling (poles and zeros) and low frequency capacity can be easily deducted to this first pole. Another advantage of Foster's models deals with a possibility to predict system response in time domain, that is difficult when Warburg impedance or constant phase element (CPE) are used for identification. The proposed approach can be generalized for different electrochemical systems and interfaces.

\section{6. $\underline{\text { References }}$}

[1] M.E. Orazem, B. Tribollet, Electrochemical Impedance Spectroscopy. John Wiley \& Sons, New Jersey, 2008.

[2] T. Pajkossy, R. Jurczakowski, Electrochemical impedance spectroscopy in interfacial studies, Current Opinion in Electrochemistry 1 (2017) 53-58. https://doi.org/10.1016/j.coelec.2017.01.006.

[3] P. Córdoba-Torres, Relationship between constant-phase element (CPE) parameters and physical properties of films with a distributed resistivity, Electrochim. Acta 225 (2017) 592-604. https://doi.org/10.1016/j.electacta.2016.12.087.

[4] C.L. Alexander, B. Tribollet, M.E. Orazem Contribution of Surface Distributions to Constant-PhaseElement (CPE) Behavior: 1. Influence of Roughness, Electrochim. Acta 173 (2015) 416-424. https://doi.org/10.1016/j.electacta.2015.05.010.

[5] C.L. Alexander, B. Tribollet, M.E. Orazem, Contribution of Surface Distributions to Constant-PhaseElement (CPE) Behavior: 2. Capacitance, Electrochim. Acta 188 (2016) 566-573. https://doi.org/10.1016/j.electacta.2015.11.135.

[6] C.L. Alexander, B. Tribollet, M.E. Orazem, Contribution of Surface Distributions to Constant-PhaseElement (CPE) Behavior: 3. Adsorbed Intermediates, Electrochim. Acta 251 (2017) 99-108. https://doi.org/10.1016/j.electacta.2017.08.081.

[7] S. Buteau, D. C. Dahn, J. R. Dahn, Explicit Conversion between Different Equivalent Circuit Models for Electrochemical Impedance Analysis of Lithium-Ion Cells, J. Electrochem. Soc. 165 (2018) A228-A234. https://doi.org/10.1149/2.0841802jes. 
[8] E. Kuhn, C. Forgez, P. Lagonotte, G. Friedrich, Modelling NI-mH battery using Cauer and Foster structures, J. Power Sources 158 (2006)1490-1497. https://doi.org/10.1016/j.jpowsour.2005.10.018.

[9] F. Soulier, P. Lagonotte, Modeling distributed parameter system with discrete element networks, $15^{\text {th }}$ Mathematical Theory of Networks and Systems, 12-16 août 2002, Notre-Dame, Indiana U.S.A.

[10] M. Chandesris, C. Robin, M. Gerard, Y. Bultel, Investigation of the difference between the low frequency limit of the impedance spectrum and the slope of the polarization curve, Electrochim. Acta 180 (2015) 581-590. https://doi.org/10.1016/j.electacta.2015.08.089.

[11] I. Pivac, F. Barbir, Inductive phenomena at low frequencies in impedance spectra of proton exchange membrane fuel cells - A review, J. Power Sources 326 (2016) 112-119. https://doi.org/10.1016/j.jpowsour.2016.06.119.

[12] J. Newman, Resistance for Flow of Current to a Disk, J. Electrochem. Soc. 5 (1966) 501-502.

[13] B. A. Boukamp, "Equivalent Circuit," Report CT88/265/128, CT89/214/128, University of Twente, The Netherlands, 1989.

[14] W. Bequette, Process Control: Modeling, Design and Simulation. Prentice Hall, New Jersey, 2002.

\section{Acknowledgments}

This work pertains to the French Government program "Investissements d'Avenir" (LABEX INTERACTIFS, reference ANR-11-LABX-0017-01). 


\section{Annexe 1: \\ Interpretation of system response in time domain using factorized expression for impedance}

Fig. A.1 demonstrates voltage response to a current step jump for $1^{\text {st }}$ order circuit presented on Fig. 6. The parameters of the circuit correspond to the ones obtained in the present study. One can see, the perfect agreement between the time-constant of the system $\tau$ and $1 / \mathrm{P} 1$ value. After a time period equivalent to 1 time constant $\tau$, the voltage reaches $63 \%$ of the final value. After a period, equivalent to $3 \tau$, $95 \%$ of the final voltage value is obtained.

\section{Annexe 2:}

\section{Results of parameter identification for $I=100 \mu \mathrm{A}$ and $\mathrm{I}=200 \mu \mathrm{A}$}

The analogous results have been obtained for impedance measurements hors the equilibrium. Table A.1 illustrates the identification of impedances data for the point $\mathrm{I}=100 \mu \mathrm{A}$.

The table A. 2 concern the identification provided for the point $\mathrm{I}=200 \mu \mathrm{A}$. 


\section{Tables}

\begin{tabular}{|l|l|}
\hline$I=0 \mu \mathrm{A}$ & $R_{d y n}=245.8 \Omega$ \\
\hline$I=100 \mu \mathrm{A}$ & $R_{d y n}=266.2 \Omega$ \\
\hline$I=200 \mu \mathrm{A}$ & $R_{d y n}=367.8 \Omega$ \\
\hline
\end{tabular}

Table 1. Dynamic resistance for specified values of the current I

\begin{tabular}{|c|c|c|c|c|c|c|}
\hline $\mathbf{0} \boldsymbol{\mu A}$ & $\mathbf{N}=\mathbf{1}$ & $\mathbf{N}=\mathbf{2}$ & $\mathbf{N}=\mathbf{3}$ & $\mathbf{N}=\mathbf{4}$ & $\mathbf{N}=\mathbf{5}$ & $\mathbf{N}=\mathbf{6}$ \\
\hline Criteria & $\mathbf{0 . 1 8 0 4}$ & $\mathbf{0 . 0 9 0 8}$ & $\mathbf{0 . 0 4 2 2}$ & $\mathbf{0 . 0 2 5 2}$ & $\mathbf{0 . 0 1 7 9}$ & $\mathbf{0 . 0 1 4 7}$ \\
\hline $\mathbf{R}_{\boldsymbol{\Sigma}}$ & $\mathbf{2 1 8 . 5 7}$ & $\mathbf{2 2 3 . 4 8}$ & $\mathbf{2 3 3 . 7 4}$ & $\mathbf{2 3 5 . 8 6}$ & $\mathbf{2 3 6 . 6 5}$ & $\mathbf{2 3 7 . 3 6}$ \\
\hline $\mathbf{R}_{\mathbf{0}}$ & $\mathbf{5 3 . 7 2}$ & $\mathbf{4 6 . 0 4}$ & $\mathbf{4 5 . 8 7}$ & $\mathbf{4 5 . 7 4}$ & $\mathbf{4 5 . 6 4}$ & $\mathbf{4 5 . 6 2}$ \\
\hline $\mathbf{C}_{\mathbf{1}}$ & $9.26 \mathrm{E}-04$ & $2.27 \mathrm{e}-06$ & $2.97 \mathrm{E}-04$ & $6.81 \mathrm{E}-04$ & $9.99 \mathrm{E}-04$ & $1.51 \mathrm{E}-03$ \\
\hline $\mathbf{R}_{\mathbf{1}}$ & 164.84 & 19.444 & 28.93 & 28.18 & 26.94 & 24.78 \\
\hline $\mathbf{C}_{\mathbf{2}}$ & & 0.00108 & $1.919 \mathrm{E}-06$ & $8.65 \mathrm{E}-05$ & $2.30 \mathrm{E}-04$ & $5.04 \mathrm{E}-04$ \\
\hline $\mathbf{R}_{\mathbf{2}}$ & & 157.99 & 16.35 & 10.93 & 11.56 & 12.66 \\
\hline $\mathbf{C}_{\mathbf{3}}$ & & & $1.637 \mathrm{E}-03$ & $1.65 \mathrm{E}-06$ & $1.82 \mathrm{E}-05$ & $1.12 \mathrm{E}-04$ \\
\hline $\mathbf{R}_{\mathbf{3}}$ & & & 142.56 & 13.57 & 7.49 & 6.65 \\
\hline $\mathbf{C}_{\mathbf{4}}$ & & & & $1.83 \mathrm{E}-03$ & $1.44 \mathrm{E}-06$ & $7.25 \mathrm{E}-06$ \\
\hline $\mathbf{R}_{\mathbf{4}}$ & & & & 137.41 & 9.92 & 7.95 \\
\hline $\mathbf{C}_{\mathbf{5}}$ & & & & & $1.93 \mathrm{E}-03$ & $1.45 \mathrm{E}-06$ \\
\hline $\mathbf{R}_{\mathbf{5}}$ & & & & & 135.07 & 6.98 \\
\hline $\mathbf{C}_{\mathbf{6}}$ & & & & & & 0.0020 \\
\hline $\mathbf{R}_{\mathbf{6}}$ & & & & & & 132.68 \\
\hline
\end{tabular}

Table 2. Identified parameters for Foster's model $(I=0 \mu A)$

\begin{tabular}{|c|c|c|c|}
\hline Point & $R_{0}(\mathrm{~N}=4)$ & $R_{\Sigma}(\mathrm{N}=4)$ & $R_{\text {dyn }}$ \\
\hline $\mathrm{I}=0 \mu \mathrm{A}$ & $\mathbf{4 5 . 7} \boldsymbol{\Omega}$ & $\mathbf{2 3 5 . 8} \boldsymbol{\mathbf { 2 4 5 . 8 }} \boldsymbol{\Omega}$ \\
\hline $\mathrm{I}=100 \mu \mathrm{A}$ & $\mathbf{4 5 . 5} \boldsymbol{\Omega}$ & $\mathbf{2 5 8 . 3} \boldsymbol{\mathbf { 2 6 6 . 2 }} \boldsymbol{\Omega}$ \\
\hline $\mathrm{I}=200 \mu \mathrm{A}$ & $\mathbf{4 5 . 4} \boldsymbol{\Omega}$ & $\mathbf{3 7 3 . 1} \boldsymbol{\Omega}$ & $\mathbf{3 6 7 . 8} \boldsymbol{\Omega}$ \\
\hline
\end{tabular}

Table 3. Identified high-frequency $R_{0}$ and total $R_{\Sigma}$ resistance compared to dynamic resistance $R_{d y n}$

\begin{tabular}{|c|c|c|c|c|c|c|}
\hline $\mathbf{0} \boldsymbol{\mu} \mathbf{A}$ & $\mathbf{N = 1}$ & $\mathbf{N = 2}$ & $\mathbf{N = 3}$ & $\mathbf{N}=\mathbf{4}$ & $\mathbf{N}=\mathbf{5}$ & $\mathbf{N}=\mathbf{6}$ \\
\hline Criteria & $\mathbf{0 . 1 8 0 4}$ & $\mathbf{0 . 0 9 0 8}$ & $\mathbf{0 . 0 4 2 2}$ & $\mathbf{0 . 0 2 5 2}$ & $\mathbf{0 . 0 1 7 9}$ & $\mathbf{0 . 0 1 4 7}$ \\
\hline $\mathbf{A}=\mathbf{R}_{\mathbf{0}}$ & $\mathbf{6 0 . 7 4}$ & $\mathbf{4 6 . 0 4}$ & $\mathbf{4 5 . 8 7 9}$ & $\mathbf{4 5 . 7 4 6}$ & $\mathbf{4 5 . 6 4 7}$ & $\mathbf{4 5 . 6 2 4}$ \\
\hline $\mathbf{P}_{\mathbf{1}}$ & $\mathbf{6 . 0 1 6}$ & $\mathbf{5 . 8 5 9}$ & $\mathbf{4 . 2 8 3}$ & $\mathbf{3 . 9 5 5}$ & $\mathbf{3 . 8 3 3}$ & $\mathbf{3 . 7 2 7}$ \\
\hline $\mathbf{Z}_{\mathbf{1}}$ & 22.18 & 19.99 & 10.76 & 9.154 & 8.545 & 7.989 \\
\hline $\mathbf{P}_{\mathbf{2}}$ & & 22600 & 116.13 & 52.04 & 37.116 & 26.588 \\
\hline $\mathbf{Z}_{\mathbf{2}}$ & & 32149 & 173.37 & 75.16 & 52.299 & 36.186 \\
\hline $\mathbf{P}_{\mathbf{3}}$ & & & 31853 & 1056.5 & 375.05 & 156.48 \\
\hline $\mathbf{Z}_{\mathbf{3}}$ & & & 43234 & 1255.4 & 447.35 & 188.61 \\
\hline $\mathbf{P}_{\mathbf{4}}$ & & & & 44485.7 & 7301.1 & 1333.9 \\
\hline $\mathbf{Z}_{\mathbf{4}}$ & & & & 57758.9 & 4506 & 1484.0 \\
\hline $\mathbf{P}_{\mathbf{5}}$ & & & & & 69882 & 17315 \\
\hline $\mathbf{Z}_{\mathbf{5}}$ & & & & & 85332 & 19879 \\
\hline $\mathbf{P}_{\mathbf{6}}$ & & & & & & 98495 \\
\hline $\mathbf{Z}_{\mathbf{6}}$ & & & & & & 114100 \\
\hline
\end{tabular}

Table 4. Poles and zeros of factorized impedance $(I=0 \mu A)$ 


\begin{tabular}{|c|c|c|c|c|c|c|}
\hline $\mathbf{1 0 0} \boldsymbol{\mu A}$ & $\mathbf{N = \mathbf { 1 }}$ & $\mathbf{N}=\mathbf{2}$ & $\mathbf{N}=\mathbf{3}$ & $\mathbf{N}=\mathbf{4}$ & $\mathbf{N}=\mathbf{5}$ & $\mathbf{N}=\mathbf{6}$ \\
\hline Criteria & $\mathbf{0 . 1 9 8 1}$ & $\mathbf{0 . 0 9 4 5}$ & $\mathbf{0 . 0 4 3 9}$ & $\mathbf{0 . 0 2 6 1}$ & $\mathbf{0 . 0 1 8 5}$ & $\mathbf{0 . 0 1 5 1}$ \\
\hline $\mathbf{A}=\mathbf{R}_{\mathbf{0}}$ & $\mathbf{5 3 . 7 3}$ & $\mathbf{4 5 . 7 7}$ & $\mathbf{4 5 . 6 2}$ & $\mathbf{4 5 . 4 9}$ & $\mathbf{4 5 . 3 8}$ & $\mathbf{4 5 . 3 7}$ \\
\hline $\mathbf{P}_{\mathbf{1}}$ & $\mathbf{6 . 7 4 4}$ & $\mathbf{4 . 5 7 3}$ & $\mathbf{4 . 4 0 1}$ & $\mathbf{4 . 0 6 9}$ & $\mathbf{3 . 9 5 4}$ & $\mathbf{3 . 8 6 0}$ \\
\hline $\mathbf{Z}_{\mathbf{1}}$ & 29.989 & 12.72 & 11.43 & 9.693 & 9.084 & 8.584 \\
\hline $\mathbf{P}_{\mathbf{2}}$ & & 327.3 & 121.3 & 54.06 & 39.16 & 29.64 \\
\hline $\mathbf{Z}_{\mathbf{2}}$ & & 562.6 & 184.6 & 79.33 & 55.95 & 40.94 \\
\hline $\mathbf{P}_{\mathbf{3}}$ & & & 29799 & 1096. & 397.7 & 181.9 \\
\hline $\mathbf{Z}_{\mathbf{3}}$ & & & 42284 & 1318 & 478.4 & 220.2 \\
\hline $\mathbf{P}_{\mathbf{4}}$ & & & & 40780 & 7761 & 1591 \\
\hline $\mathbf{Z}_{\mathbf{4}}$ & & & & 55109 & 8956 & 1777 \\
\hline $\mathbf{P}_{\mathbf{5}}$ & & & & & 65166 & 18933 \\
\hline $\mathbf{Z}_{\mathbf{5}}$ & & & & & 81704 & 22374 \\
\hline $\mathbf{P}_{\mathbf{6}}$ & & & & & & 97316 \\
\hline $\mathbf{Z}_{\mathbf{6}}$ & & & & & & 113558 \\
\hline
\end{tabular}

Table 5. Poles and zeros of factorized impedance $(I=100 \mu \mathrm{A})$

\begin{tabular}{|c|c|c|c|c|c|c|}
\hline $\mathbf{2 0 0} \boldsymbol{\mu A}$ & $\mathbf{N}=\mathbf{1}$ & $\mathbf{N}=\mathbf{2}$ & $\mathbf{N}=\mathbf{3}$ & $\mathbf{N}=\mathbf{4}$ & $\mathbf{N}=\mathbf{5}$ & $\mathbf{N}=\mathbf{6}$ \\
\hline Criteria & $\mathbf{0 . 2 4 7 9}$ & $\mathbf{0 . 1 0 9 6}$ & $\mathbf{0 . 0 4 9 5}$ & $\mathbf{0 . 0 2 8 8}$ & $\mathbf{0 . 0 1 9 8}$ & $\mathbf{0 . 0 1 5 7}$ \\
\hline $\mathbf{A}=\mathbf{R}_{\mathbf{0}}$ & $\mathbf{5 2 . 1 6}$ & $\mathbf{4 5 . 6 9}$ & $\mathbf{4 5 . 5 7}$ & $\mathbf{4 5 . 4 4}$ & $\mathbf{4 5 . 3 1}$ & $\mathbf{4 5 . 2 7}$ \\
\hline $\mathbf{P}_{\mathbf{1}}$ & $\mathbf{7 . 0 3 2}$ & $\mathbf{6 . 0 5 6}$ & $\mathbf{4 . 4 7 0}$ & $\mathbf{4 . 1 5 0}$ & $\mathbf{4 . 0 1 2}$ & $\mathbf{3 . 9 3 0}$ \\
\hline $\mathbf{Z}_{\mathbf{1}}$ & 45.81 & 27.096 & 13.37 & 11.20 & 10.28 & 9.757 \\
\hline $\mathbf{P}_{\mathbf{2}}$ & & 16713 & 128.8 & 59.20 & 40.80 & 32.88 \\
\hline $\mathbf{Z}_{\mathbf{2}}$ & & 28776 & 216.2 & 94.82 & 62.61 & 48.98 \\
\hline $\mathbf{P}_{\mathbf{3}}$ & & & 22199 & 1160 & 377.4 & 212.6 \\
\hline $\mathbf{Z}_{\mathbf{3}}$ & & & 35846 & 1463 & 479.1 & 270.3 \\
\hline $\mathbf{P}_{\mathbf{4}}$ & & & & 30503 & 6678 & 1893 \\
\hline $\mathbf{Z}_{\mathbf{4}}$ & & & & 45951 & 8137 & 2179 \\
\hline $\mathbf{P}_{\mathbf{5}}$ & & & & & 47902 & 18477 \\
\hline $\mathbf{Z}_{\mathbf{5}}$ & & & & & 65119 & 23985 \\
\hline $\mathbf{P}_{\mathbf{6}}$ & & & & & & 87066 \\
\hline $\mathbf{Z}_{\mathbf{6}}$ & & & & & & 102797 \\
\hline
\end{tabular}

Table 6. Poles and zeros of factorized impedance $(I=200 \mu \mathrm{A})$

\begin{tabular}{|c|c|c|c|c|c|c|}
\hline Point & $R_{0}(N=4)$ & $\mathbf{R}_{\Sigma}(\mathbf{N}=\mathbf{4})$ & $P_{1}(N=4)$ & $\tau_{1}=1 / \mathbf{P}_{1}=\mathbf{R}_{1}^{\prime} . \mathrm{C}^{\prime}{ }_{1}$ & $\mathbf{R}^{\prime}{ }_{1}=\mathbf{R}_{\Sigma}-\mathbf{R}_{\mathbf{0}}$ & $C^{\prime}{ }_{1}=1 /\left(P_{1 . R}{ }_{1}\right)$ \\
\hline $\mathrm{I}=\mathbf{0} \boldsymbol{\mu} \mathrm{A}$ & $45.74 \Omega$ & $235.86 \Omega$ & $3.955 \mathrm{~s}^{-1}$ & 0,252 second & $190,12 \Omega$ & $1.33 .10^{-3} \mathrm{~F}$ \\
\hline $\mathrm{I}=100 \mu \mathrm{A}$ & $45.49 \Omega$ & $258.34 \Omega$ & $4.069 \mathrm{~s}^{-1}$ & 0,245 second & $212,85 \Omega$ & $1.15 .10^{-3} \mathrm{~F}$ \\
\hline$I=200 \mu A$ & $45.44 \Omega$ & $373.12 \Omega$ & $4.150 \mathrm{~s}^{-1}$ & 0,240 second & $327,68 \Omega$ & $0.73 .10^{-3} \mathrm{~F}$ \\
\hline
\end{tabular}

Table 7. Estimation of characteristic capacities of solid liquid interface

\begin{tabular}{|c|c|c|}
\hline Point & $\tau_{\mathbf{1}}=\mathbf{1} / \mathbf{P}_{\mathbf{1}}=\mathbf{R}^{\prime}{ }_{1} . \mathbf{C}^{\prime}{ }_{\mathbf{1}}$ & $\mathbf{F}_{\mathbf{c} \mathbf{1}}=\mathbf{1} /\left(2 \pi . \tau_{1}\right)$ \\
\hline $\mathbf{I}=\mathbf{0} \boldsymbol{\mu} \mathbf{A}$ & 0,252 second & $\mathbf{0 , 6 3 1} \mathbf{~ H z}$ \\
\hline $\mathbf{I}=\mathbf{1 0 0} \boldsymbol{\mu} \mathbf{A}$ & 0,245 second & $\mathbf{0 , 6 4 9} \mathbf{~ H z}$ \\
\hline $\mathbf{I}=\mathbf{2 0 0} \boldsymbol{\mu} \mathbf{A}$ & 0,240 second & $\mathbf{0 , 6 6 3} \mathbf{~ H z}$ \\
\hline
\end{tabular}

Table 8. Estimation of first time constant $\tau_{1}$ and first frequency $F_{c l}$ 


\begin{tabular}{|c|c|c|c|c|c|c|}
\hline $\mathbf{1 0 0} \boldsymbol{\mu} \mathbf{A}$ & $\mathbf{N}=\mathbf{1}$ & $\mathbf{N}=\mathbf{2}$ & $\mathbf{N}=\mathbf{3}$ & $\mathbf{N = 4}$ & $\mathbf{N}=\mathbf{5}$ & $\mathbf{N}=\mathbf{6}$ \\
\hline Criteria & $\mathbf{0 . 1 9 8 1}$ & $\mathbf{0 . 0 9 4 5}$ & $\mathbf{0 . 0 4 3 9}$ & $\mathbf{0 . 0 2 6 1}$ & $\mathbf{0 . 0 1 8 5}$ & $\mathbf{0 . 0 1 5 1}$ \\
\hline $\mathbf{R}_{\boldsymbol{\Sigma}}$ & $\mathbf{2 3 8 . 9 2}$ & $\mathbf{2 4 4 . 8 8}$ & $\mathbf{2 5 5 . 9 5}$ & $\mathbf{2 5 8 . 3 4}$ & $\mathbf{2 5 9 . 1 7}$ & $\mathbf{2 5 9 . 8 4}$ \\
\hline $\mathbf{R}_{\mathbf{0}}$ & $\mathbf{5 3 . 7 3}$ & $\mathbf{4 5 . 7 7}$ & $\mathbf{4 5 . 6 2}$ & $\mathbf{4 5 . 4 9}$ & $\mathbf{4 5 . 3 8}$ & $\mathbf{4 5 . 3 7}$ \\
\hline $\mathbf{C}_{\mathbf{1}}$ & $8.00 \mathrm{E}-04$ & $2.02 \mathrm{E}-06$ & $2.59 \mathrm{E}-04$ & $5.97 \mathrm{E}-04$ & $8.66 \mathrm{E}-04$ & $1.23 \mathrm{E}-03$ \\
\hline $\mathbf{R}_{\mathbf{1}}$ & 185.18 & 22.50 & 31.76 & 30.92 & 29.47 & 27.30 \\
\hline $\mathbf{C}_{\mathbf{2}}$ & & $9.48 \mathrm{E}-04$ & $1.75 \mathrm{E}-06$ & $7.50 \mathrm{E}-05$ & $1.98 \mathrm{E}-04$ & $4.07 \mathrm{E}-04$ \\
\hline $\mathbf{R}_{\mathbf{2}}$ & & 176.60 & 19.07 & 12.15 & 12.63 & 13.48 \\
\hline $\mathbf{C}_{\mathbf{3}}$ & & & $1.42 \mathrm{E}-03$ & $1.54 \mathrm{E}-06$ & $1.45 \mathrm{E}-05$ & $8.78 \mathrm{E}-05$ \\
\hline $\mathbf{R}_{\mathbf{3}}$ & & & 159.49 & 15.88 & 8.87 & 7.15 \\
\hline $\mathbf{C}_{\mathbf{4}}$ & & & & $1.59 \mathrm{E}-03$ & $1.36 \mathrm{E}-06$ & $5.37 \mathrm{E}-06$ \\
\hline $\mathbf{R}_{\mathbf{4}}$ & & & & 153.88 & 11.26 & 9.81 \\
\hline $\mathbf{C}_{\mathbf{5}}$ & & & & & $1.66 \mathrm{E}-03$ & $1.42 \mathrm{E}-06$ \\
\hline $\mathbf{R}_{\mathbf{5}}$ & & & & & 151.53 & 7.22 \\
\hline $\mathbf{C}_{\mathbf{6}}$ & & & & & & $1.73 \mathrm{E}-03$ \\
\hline $\mathbf{R}_{\mathbf{6}}$ & & & & & & 149.49 \\
\hline
\end{tabular}

Table A.1. Identified parameters for Foster's model $(I=100 \mu A)$

\begin{tabular}{|c|c|c|c|c|c|c|}
\hline $\mathbf{2 0 0} \boldsymbol{\mu A}$ & $\mathbf{N = 1}$ & $\mathbf{N = 2}$ & $\mathbf{N = 3}$ & $\mathbf{N}=\mathbf{4}$ & $\mathbf{N}=\mathbf{5}$ & $\mathbf{N}=\mathbf{6}$ \\
\hline $\mathbf{C r i t e r i a}$ & $\mathbf{0 . 2 4 7 9}$ & $\mathbf{0 . 1 0 9 6}$ & $\mathbf{0 . 0 4 9 5}$ & $\mathbf{0 . 0 2 8 8}$ & $\mathbf{0 . 0 1 9 8}$ & $\mathbf{0 . 0 1 5 7}$ \\
\hline $\mathbf{R}_{\boldsymbol{\Sigma}}$ & $\mathbf{3 3 9 . 8 6}$ & $\mathbf{3 5 1 . 9 7}$ & $\mathbf{3 6 9 . 6 6}$ & $\mathbf{3 7 3 . 1 2}$ & $\mathbf{3 7 4 . 7 1}$ & $\mathbf{3 7 5 . 5 9}$ \\
\hline $\mathbf{R}_{\mathbf{0}}$ & $\mathbf{5 2 . 1 6}$ & $\mathbf{4 5 . 6 9}$ & $\mathbf{4 5 . 5 7}$ & $\mathbf{4 5 . 4 4}$ & $\mathbf{4 5 . 3 1}$ & $\mathbf{4 5 . 2 7}$ \\
\hline $\mathbf{C}_{\mathbf{1}}$ & $4.94 \mathrm{E}-04$ & $1.81 \mathrm{E}-06$ & $1.67 \mathrm{E}-04$ & $3.68 \mathrm{E}-04$ & $5.64 \mathrm{E}-04$ & $7.34 \mathrm{E}-04$ \\
\hline $\mathbf{R}_{\mathbf{1}}$ & 287.69 & 32.93 & 46.46 & 45.82 & 43.39 & 41.38 \\
\hline $\mathbf{C}_{\mathbf{2}}$ & & $6.04 \mathrm{E}-04$ & $1.61 \mathrm{E}-06$ & $4.94 \mathrm{E}-05$ & $1.40 \mathrm{E}-04$ & $2.40 \mathrm{E}-04$ \\
\hline $\mathbf{R}_{\mathbf{2}}$ & & 273.34 & 27.89 & 17.42 & 18.83 & 19.56 \\
\hline $\mathbf{C}_{\mathbf{3}}$ & & & $8.95 \mathrm{E}-04$ & $1.44 \mathrm{E}-06$ & $1.08 \mathrm{E}-05$ & $5.12 \mathrm{E}-05$ \\
\hline $\mathbf{R}_{\mathbf{3}}$ & & & 249.73 & 22.74 & 13.75 & 10.31 \\
\hline $\mathbf{C}_{\mathbf{4}}$ & & & & $9.96 \mathrm{E}-04$ & $1.33 \mathrm{E}-06$ & $3.33 \mathrm{E}-06$ \\
\hline $\mathbf{R}_{\mathbf{4}}$ & & & & 241.68 & 15.66 & 16.23 \\
\hline $\mathbf{C}_{\mathbf{5}}$ & & & & & $1.04-03$ & $1.53 \mathrm{E}-06$ \\
\hline $\mathbf{R}_{\mathbf{5}}$ & & & & & 237.74 & 7.49 \\
\hline $\mathbf{C}_{\mathbf{6}}$ & & & & & & $1.08 \mathrm{E}-03$ \\
\hline $\mathbf{R}_{\mathbf{6}}$ & & & & & & 235.32 \\
\hline
\end{tabular}

Table A.2. Identified parameters for Foster's model $(I=200 \mu \mathrm{A})$

\section{Figures Captions}

Fig. 1. Foster's series models representation for different stages

Fig. 2. Voltage-current curve of ferri-ferro solution

Fig. 3. Impedance curves for $0 \mu \mathrm{A}, 100 \mu \mathrm{A}, 200 \mu \mathrm{A}$

Fig. 4. Results of fitting for $0 \mu A, 100 \mu A, 200 \mu A$ and $N=4$

Fig. 5. Developed and factorized Foster's impedance

Fig. 6. Simplified model with one pole and one zero.

Fig. 7. Comparison in the Nyquist diagram between $Z_{\text {foster }}$ and $Z_{\text {factorised }}$ for $I=0 \mu \mathrm{A} I=100 \mu \mathrm{A}, I=200 \mu \mathrm{A}$ (Foster's model $N=4$ with 9 parameters in blue, Factorised model with 3 parameters in red)

Fig. A.1. Example of step response for $1^{\text {st }}$ time constant model 


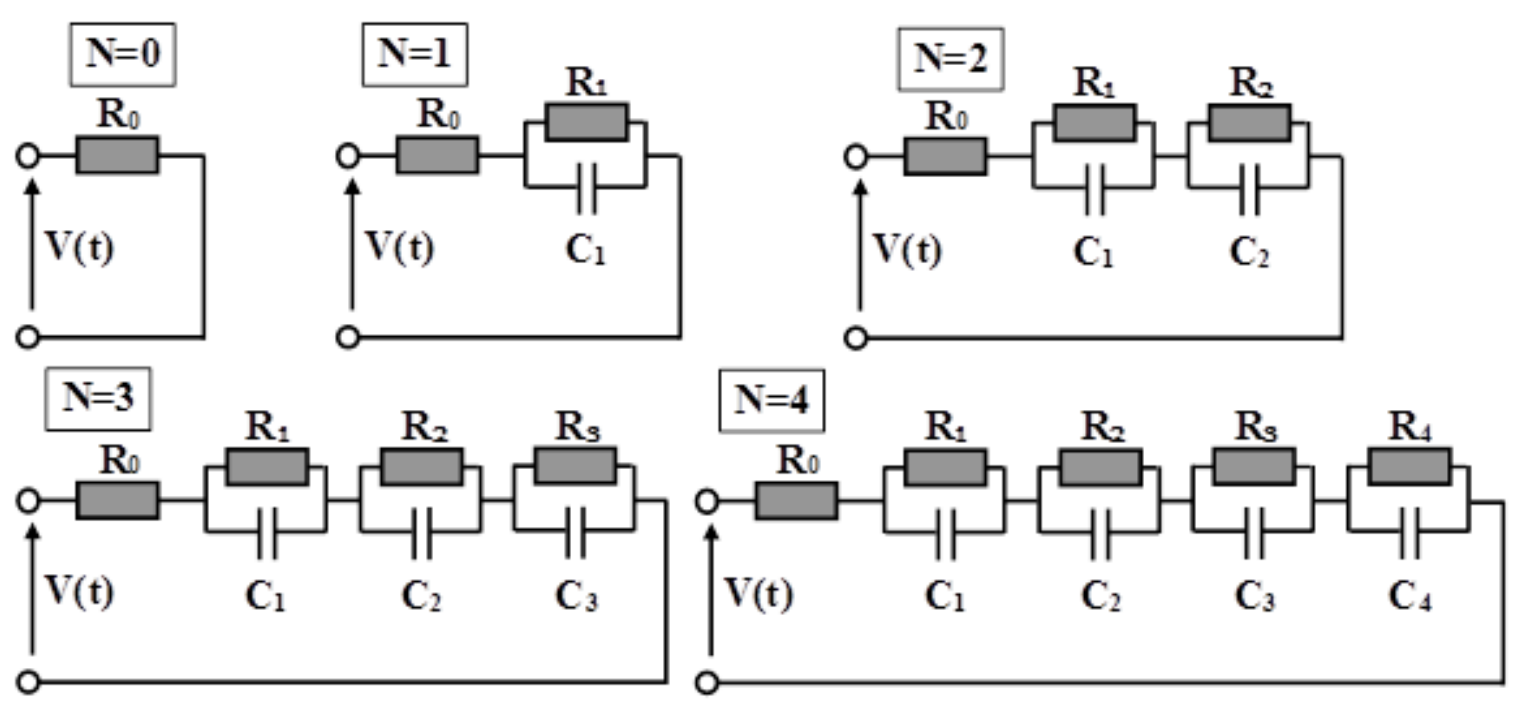


Voltage-current curve

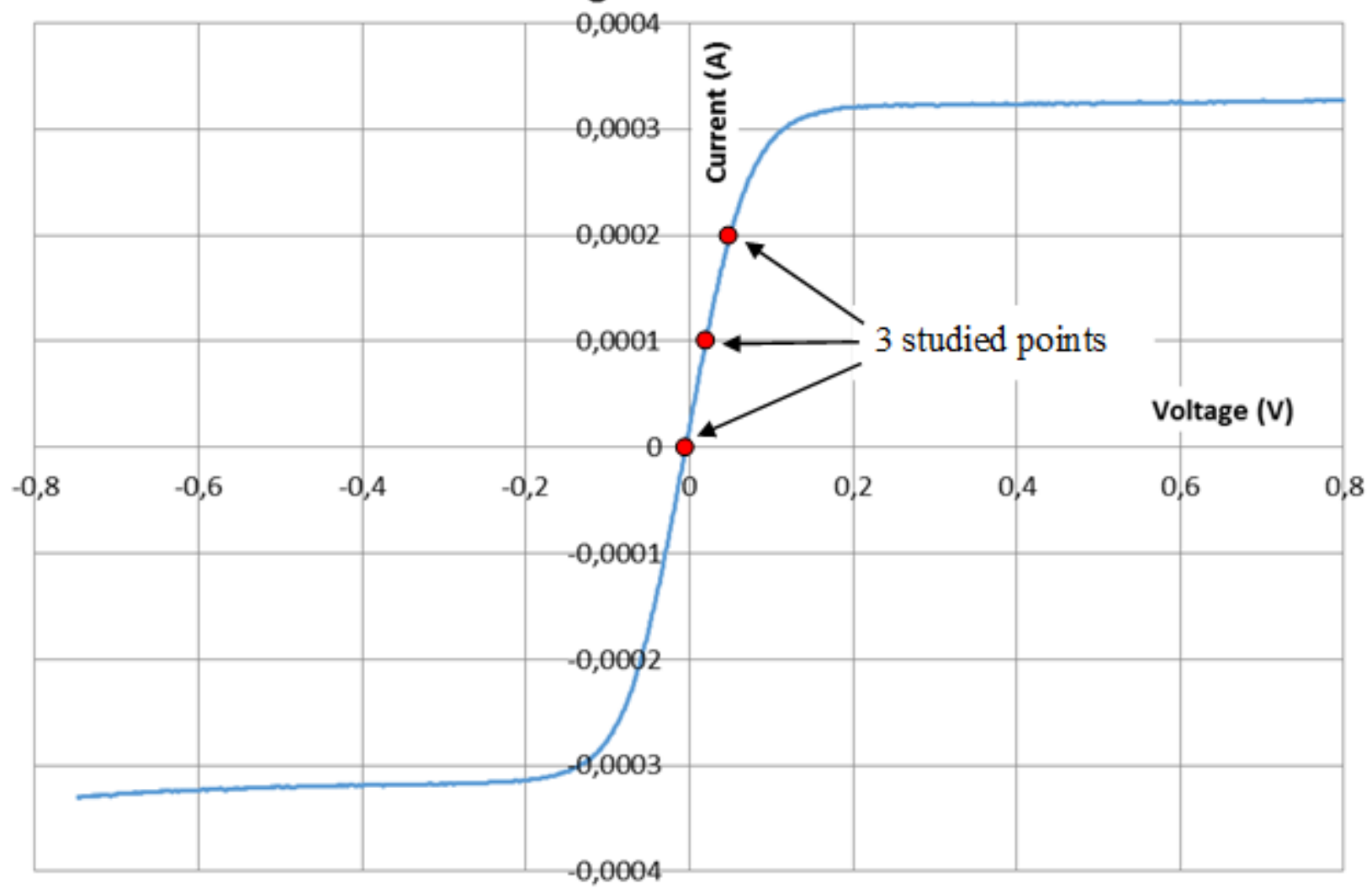




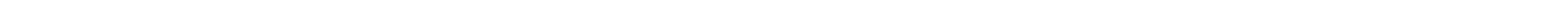


Foster's model

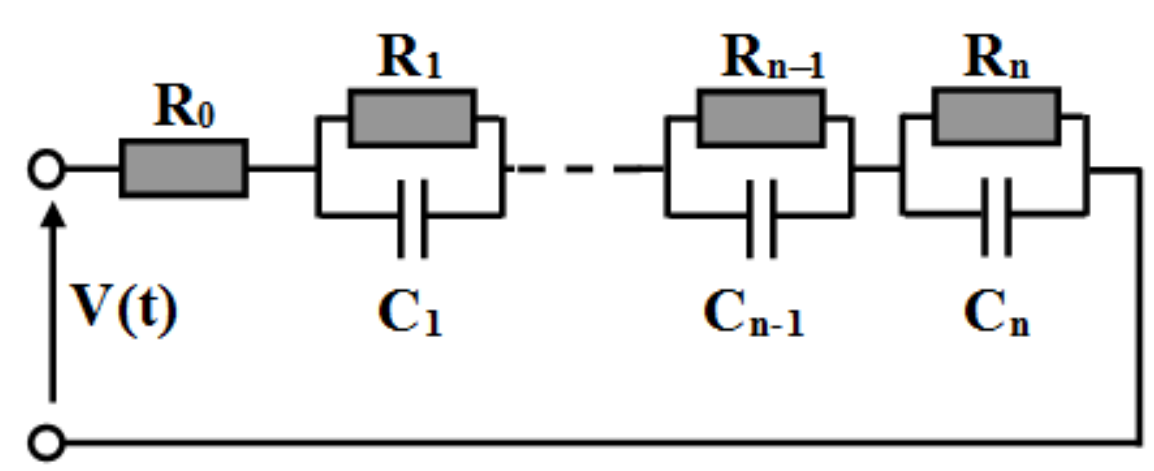

Developed Foster's Impedance

$$
Z_{\text {Foster }}=R_{0}+\frac{1}{s \cdot C_{1}+\frac{1}{R_{1}}}+\cdots+\frac{1}{s \cdot C_{n}+\frac{1}{R_{n}}}
$$

Factorised Foster's Impedance

$$
Z_{\text {Foster }}=\frac{A\left(s+Z_{1}\right) \cdots\left(s+Z_{n}\right)}{\left(s+P_{1}\right) \cdots\left(s+P_{n}\right)}
$$




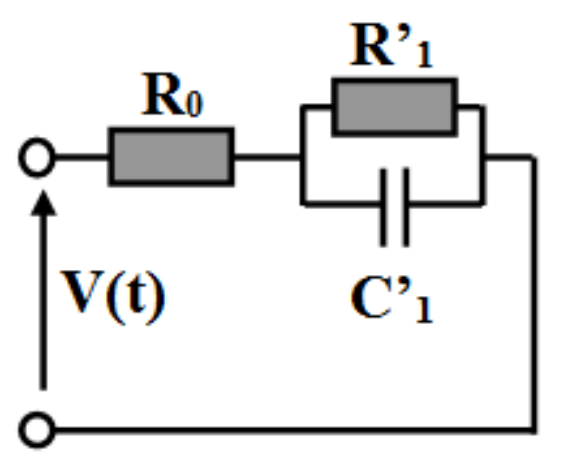

Developed Foster's Impedance

$$
Z=R_{0}+\frac{1}{s C^{\prime}{ }_{1}+\frac{1}{R_{1}^{\prime}}}
$$

Factorised Foster's Imped ance

$$
Z=\frac{R_{0}\left(s+\frac{R_{0}+R_{1}^{\prime}}{R_{0} R_{1}^{\prime} C_{1}^{\prime}}\right)}{\left(s+\frac{1}{C_{1}^{\prime} R_{1}^{\prime}}\right)}=\frac{R_{0}\left(s+Z_{1}\right)}{\left(s+P_{1}\right)}
$$


